\title{
Game theory approach on sustainable tourism
}

\author{
R. Mosetti \\ Istituto Nazionale di Oceanografia e di Geofisica Sperimentale, Trieste, \\ Italy
}

\begin{abstract}
A two agents non-cooperative model is applied in the context of tourism. It is shown that a sharing of resources between the public and private sector gives an optimal solution which is stable in the sense that for both agents the deviation from the optimal solution is not convenient. The optimal solution corresponds to the social optimum.

Keywords: sustainable tourism, non-cooperative game theory.
\end{abstract}

\section{Introduction}

Tourism is a typical industry in which externalities are present due to the access to common resources (Tyrrell [1], Sinclair [2]). The problem is under the so-called "tragedy of commons" (Hardin [3], Ostrom [4]) in which each economic subject is trying to maximise his own profit from common resources like the environment. Action has to be undertaken by the public sector (local, regional or national authorities) in order to re-allocate the non-optimal equilibrium arising from such externalities. This paper is a short note concerning the application of game theory to a situation in which the private sector and the public one are the agents in a non-cooperative game. Some application of game theory to social conflicts is reported for instance in Binmore [5].

\section{A two-agent model}

A two agents model is considered to investigate the optimal solutions. The first actor is the private sector, the second the public services. 


$$
\begin{gathered}
\Pi=p F(q)-C(q, \omega) \\
S=p \beta \omega-D(q, \omega)
\end{gathered}
$$

where:

$\mathrm{q}$ is the number of tourists; $\omega$ are the expenditures by the public sector (investments + social costs).

$\Pi$ is the net profit of the private sector from tourism; $\mathrm{C}(.,$.$) is the costs for$ producing $F(q)$ depending also on $\omega$ (e.g. tax wages ).

$\mathbf{S}$ is the public sector benefit including the social costs $\mathrm{D}(.,$.$) depending on the$ size of the tourism pressure and on expenditures. $\beta$ is the fraction of $\omega$ returning as income for instance from taxation.

The first order conditions (i.e. optimisation of each agent utility without taking into account the other factor) would give:

$$
\begin{gathered}
\frac{\partial \Pi}{\partial q}=p F^{\prime}(q)-\frac{\partial C}{\partial q}=0 \Rightarrow p F^{\prime}(q)=\frac{\partial C}{\partial q} \\
\frac{\partial S}{\partial \omega}=p \beta-\frac{\partial D}{\partial \omega}=0 \Rightarrow p \beta=\frac{\partial D}{\partial \omega}
\end{gathered}
$$

Now let us consider the social utility given as usual by:

$$
\Pi^{\prime}=\Pi+S
$$

so that the optimal value for $\mathrm{q}$ and $\omega$ are obtained by solving:

$$
\begin{aligned}
& \frac{\partial \Pi^{\prime}}{\partial q}=p F^{\prime}(q)-\left[\frac{\partial C}{\partial q}+\frac{\partial D}{\partial q}\right]=0 \\
& \frac{\partial \Pi^{\prime}}{\partial \omega}=p \beta-\left[\frac{\partial C}{\partial \omega}+\frac{\partial D}{\partial \omega}\right]=0
\end{aligned}
$$

The optimal values $\left(q^{*}, \omega^{*}\right)$ obtained by Eqs. (2) are of course different from those obtained from the first order conditions (1). The equilibrium is a Pareto optimum. Now a key question is:

What happens if one agent moves from the social optimal value $\left(q^{*}, \omega^{*}\right)$ trying to increase its own profit?

Non-cooperative game theory ( see for example Gibbons [6]) will help us in order to tackle the problem. 
Let's consider the following pay-off matrix (Prisoner's dilemma):

$$
\begin{array}{ccc}
\Pi / S & \text { Cooperate } & \text { Defect } \\
\text { Cooperate } & \left(q^{*}, \omega^{*}\right) & (+,-) \\
\text { Defect } & (-,+) & (-,-)
\end{array}
$$

where + indicates an increasing of the profit, while - indicates a decreasing. For instance (+,-) means that agent $\Pi$ defecting while $S$ stay on the social optimum increase its profit causing a decrease of the utlity of $\mathrm{S}$. From this it is evident that there is a clear incentive to defect for both agents. This is a typical situation also in environmental problems related for instance in the $\mathrm{CO} 2$ emission treaties. The situation (-,-) is a Nash equilibrium for the non-cooperative game and it constitutes a much strong condition than a paretian optimum. It is clear that this solution is an incentive to defect.

To overcame the problem of the incentivation of defecting, a shared profit problem can be posed. This approach has been established for instance in partial equilibrium models of externalities in firm interacting production problem, (Runge [7], Varian [8]). Let's considered, for the private and public sectors the following situation of mixed capitals:

$$
\begin{gathered}
\Pi^{s}=u \Pi+(1-v) S \\
(0<u<1 ; 0<v<1) \\
S^{s}=(1-u) \Pi+v S
\end{gathered}
$$

The meaning is that both the private and public sector will share their "profits" (measured by $u$ and $v$ ) among them. This can be done for example by the participation of public capitals into private holdings.

The optimum for the private sector is given by solving:

$$
\frac{\partial \Pi^{S}}{\partial q}=u p F^{\prime}(q)-\left[u \frac{\partial C}{\partial q}+(1-v) \frac{\partial D}{\partial q}\right]=0
$$

All the mathematical results depends on the assumption made for the structure of the cost and production function such as: continuity, convexity, existence of extrema. We will assume in the following that functions are "good functions".

The first condition gives:

$$
p F^{\prime}(q)=\left[\frac{\partial C}{\partial q}+\frac{1-v}{u} \frac{\partial D}{\partial q}\right]
$$


Now, if $u=v=1 / 2$ (symmetric generosity rule) the individual optimum given by (4) is coincident with the social optimum from Eq. (2). The same for the public sector $\mathrm{S}$. Thus, the equilibrium solution is $\left(q^{*}, \omega^{*}\right)$. What happen in this case to the robustness of the equilibrium against incentives to defect? Let's go to the pay-off matrix. In this case:

$$
\begin{array}{ccc}
\Pi / S & \text { Cooperate } & \text { defect } \\
\text { Cooperate } & \left(q^{*}, \omega^{*}\right) & (-,-) \\
\text { Defect } & (-,-) & (-,-)
\end{array}
$$

There is no incentive to defect. The equilibrium $\left(q^{*}, \omega^{*}\right)$ is stable.

\section{Conclusions}

The idea here expressed, even through a naïve model, is that by sharing the resources of public and private sectors in the tourism industry, could be a way for sustaining the social optimum.

\section{References}

[1] Tyrrel, T. (1998) “Elements of Sustainable Tourism” in: Proceedings of the Third Conference on Tourism and Hotel Industry in Ido-China and South-East Asia. Price of Sonkla University, Phuket, Thailand: 29-34.

[2] Sinclair, M. T. (1991) The Economics of Tourism. Progress in Tourism, Recreation and Hospitality Management, Vo. 3, pp. 1-27.

[3] Hardin, G. (1968) The Tragedy of commons, Science 162: 1234-1238.

[4] Ostrom, E. (1990) Governing the Commons: the Evolution of Institutions for Collective Action. Cambridge University Press, New York.

[5] Binmore, K. (1998) Game Theory and Social Contract. MIT Press, Cambridge, Massachusetts.

[6] Gibbons, R. (1992) A Primer on Game Theory. Harvester Wheatsheaf.

[7] Runge, F.C. (1981). Common property externalities: isolation, assurance, and resource depletion in a traditional grazing context. American Journal of Agricultural Economics 63:595-606.

[8] Varian, Hal R. 1994. A solution to the problem of externalities when agents are well-informed. American Economic Review 84(5): 1278-1293. 